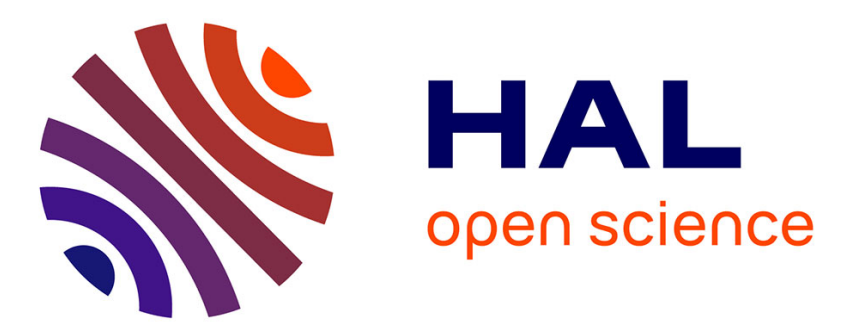

\title{
Assessment of a numerical method for computing the spherical harmonic coefficients of the gravitational potential of a constant density polyhedron
}

O. Jamet, Jérôme Verdun, D Tsoulis, N Gonindard

\section{- To cite this version:}

O. Jamet, Jérôme Verdun, D Tsoulis, N Gonindard. Assessment of a numerical method for computing the spherical harmonic coefficients of the gravitational potential of a constant density polyhedron. IAG International Symposium GGEO2008, Jun 2008, Chania, Greece. 10.1007/978-3-642-10634-7_58 . hal-02127812

\section{HAL Id: hal-02127812 \\ https://hal.science/hal-02127812}

Submitted on 13 May 2019

HAL is a multi-disciplinary open access archive for the deposit and dissemination of scientific research documents, whether they are published or not. The documents may come from teaching and research institutions in France or abroad, or from public or private research centers.
L'archive ouverte pluridisciplinaire HAL, est destinée au dépôt et à la diffusion de documents scientifiques de niveau recherche, publiés ou non, émanant des établissements d'enseignement et de recherche français ou étrangers, des laboratoires publics ou privés. 


\title{
Assessment of a numerical method for computing the spherical harmonic coefficients of the gravitational potential of a constant density polyhedron
}

\author{
O. Jamet ${ }^{1}$, J. Verdun ${ }^{1,2}$, D. Tsoulis ${ }^{3}$, and N. Gonindard ${ }^{2}$ \\ nov. 2008
}

\begin{abstract}
${ }^{1}$ Laboratoire de Recherche en Géodesie, IGN, 2 av. Pasteur, 94160 Saint-Mandé, France, email: \{Olivier.Jamet,Jerome.Verdun\}@ign.fr

${ }^{2}$ Ecole Nationale des Sciences Geographiques, 6 et 8 av. Blaise Pascal, Cite Descartes, 77455 Marne-La-Vallee CEDEX, France

${ }^{3}$ Department of Geodesy and Surveying, Aristotle University of Thessaloniki, 54124 Thessaloniki, Greece, email: tsoulis@topo.auth.gr
\end{abstract}

\begin{abstract}
This study focuses on the assessment of a linear algorithm for computing the spherical harmonic coefficients of the gravitational potential of a constant density polyhedron. The ability to compute such an expansion would favor several applications, in particular in the field of the interpretation and assessment of GOCE gravitational models. The studied algorithm is the only known method that would achieve this computation at a computational cost depending linearly on the number of computed coefficients. We show that although this methods suffers from severe divergence issues, it could be applied to retrieve band-limited estimates of the potential generated by a constant density polyhedron.
\end{abstract}

Keywords. Gravitational potential, polyhedron, spherical harmonics

\section{Introduction}

Constant density polyhedra provide a versatile representation of whole planetary bodies as well as density discontinuities inside a planet at any scale. This representation allows the exact computation of the gravitational potential of 
such features through well known analytical formulas (Barnett, 1976; Petrović, 1996; Pohánka, 1988). These formulas yield the potential value at any computation point, providing that their singularities are properly managed (Tsoulis and Petrović, 2001). Applications range from the accurate computation of the terrain effect (Tsoulis, 2001) to the modelling of the gravitational field of small planetary bodies (Simonelli et al., 1993; Werner and Scheeres, 1996). Such analytical computations show two major drawbacks. First, the computation is pointwise: one single computation yields the potential or its directional derivatives at a single computation point. The computation might become highly tedious as the number of computing locations increases. Secondly, in the context of some specific applications, one might seek for a band-limited estimate of the potential, which cannot be obtained through these analytical formulas.

In the context of the forthcoming GOCE satellite, we are here interested in numerical methods for computing the spherical harmonic expansion of the gravitational potential of a constant density polyhedron. A direct computation of such an expansion from the geometry of the polyhedron would provide on the one hand a band-limited estimate of the potential that would be directly comparable to GOCE derived potential models, and on the other hand a fast access to the value of this band-limited potential at a large number of locations. Such a tool would enhance the interpretation of GOCE data, allowing to remove the signal from known features (topography, local geological models, etc.) from GOCE derived gravitational models at chosen wavelengths. When the density of the body can be described as a function of the direct distance to its geometric center, the computation of the spherical harmonic expansion of its potential admits some numerical (Chao and Rubincam, 1989) or even analytical (Balmino, 1994; Martinec et al., 1989) solutions. In the case of the general polyhedron, no closed formulation is known. We are aware of two numerical methods, proposed by Werner (1997), and Jamet and Thomas (2004). Both rely on recursion relationships. The quadratic complexity of Werner's method makes it only applicable to very low degrees. The method by Jamet and Thomas is of linear complexity with respect to the number of computed coefficients and the number of edges of the polyhedron. It has never been numerically implemented yet. We focus here on the assessment of Jamet and Thomas's algorithm. The implementation was adapted in order to work with fully normalized spherical harmonics, as defined by Heiskanen and Moritz (1967, p. 31). In section 2, we restrict ourselves to a brief presentation of the principles of the method. Then, in section 3, we present some numerical experiments that underline the strenghts and weaknesses of the proposed method. Finally, in section 4, we comment on the possible applications of this algorithm.

\section{Algorithm}

The spherical harmonic expansion of the gravitational potential of any finite body can be written as

$$
V(r, \theta, \lambda)=\frac{G M}{r}\left\{1+\sum_{n=1}^{+\infty} \sum_{m=0}^{n} V_{n, m}(r, \theta, \lambda)\right\}
$$


with

$$
\begin{aligned}
V_{n, m}(r, \theta, \lambda)= & c_{n, m} R_{n, m}(r, \theta, \lambda)+ \\
& s_{n, m} S_{n, m}(r, \theta, \lambda)
\end{aligned}
$$

where $(r, \theta, \lambda)$ are the spherical coordinates of the considered point (radius, colatitude and longitude), $G$ the gravitational constant, $M$ the mass of the body, $R_{n, m}$ and $S_{n, m}$ are the irregular solid harmonics, defined here as

$$
\begin{aligned}
R_{n, m}(r, \theta, \lambda) & =\left(\frac{a}{r}\right)^{n} P_{n, m}(\cos \theta) \cos (m \lambda) \\
S_{n, m}(r, \theta, \lambda) & =\left(\frac{a}{r}\right)^{n} P_{n, m}(\cos \theta) \sin (m \lambda)
\end{aligned}
$$

with $a$ being an arbitrary length greater than the longest radius including the attracting sources. In this expression, $c_{n, m}$ and $s_{n, m}$ are the coefficients of the expansion to be computed. These coefficients, like their normalized counterparts, can be written as a volume integral defined over the whole body (Heiskanen and Moritz, 1967). When the body is of constant density, this volume integral writes

$$
\begin{aligned}
& c_{n, m}=k_{n, m}^{(c)} \iiint_{Q \in b o d y} h_{n, m}^{(c)}(Q) d v \\
& s_{n, m}=k_{n, m}^{(s)} \iiint_{Q \in b o d y} h_{n, m}^{(s)}(Q) d v
\end{aligned}
$$

where $d v$ is the volume element, $h_{n, m}^{(c)}$ and $h_{n, m}^{(s)}$ are the regular solid harmonics

$$
\begin{aligned}
& h_{n, m}^{(c)}(r, \theta, \lambda)=\left(\frac{r}{a}\right)^{n} P_{n, m}(\cos \theta) \cos (m \lambda) \\
& h_{n, m}^{(s)}(r, \theta, \lambda)=\left(\frac{r}{a}\right)^{n} P_{n, m}(\cos \theta) \sin (m \lambda)
\end{aligned}
$$

while $k_{n, m}^{(c)}$ and $k_{n, m}^{(s)}$ are constants depending on the degree $n$, the order $m$ and the density of the body. Jamet and Thomas's method relies on the following principles. Firstly, the polyhedron is decomposed into simplices originating at the origin of the coordinate system. The volume integral over each simplex is then converted into a surface integral over the corresponding planar face of the polyhedron by using the divergence theorem of Gauss. So far, this procedure is much similar to the methods for exhibiting analytical formulas of the potential, as the one used for instance by Petrović (1996). Applying the Stokes theorem to the surface integral yields then a recurrent relationship between the surface integrals of same order $m$ and of consecutive degree $n$, from which one gets

$$
\begin{aligned}
c_{n, m}= & \alpha_{n, m} c_{n-1, m}+ \\
& \sum_{e \in \text { edges }} \beta_{n, m}^{e} \int_{Q \in e} h_{n, m}^{(c)}(Q) d l \\
s_{n, m}= & \alpha_{n, m} s_{n-1, m}+ \\
& \sum_{e \in \text { edges }} \beta_{n, m}^{e} \int_{Q \in e} h_{n, m}^{(s)}(Q) d l
\end{aligned}
$$


where the line integrals are computed along each edge of this planar face, and where $\alpha_{n, m}$ and $\beta_{n, m}^{e}$ are constants depending on geometry of the handled planar face, its edges, and on degree $n$ and order $m$. In particular Jamet and Thomas (2004) show that

$$
\alpha_{n, m}=A(n, m) \frac{d}{\mathbf{u}_{z} \cdot \mathbf{n}}
$$

where $A(n, m)$ is a constant depending on degree and order, $d$ is the distance from the origin of the frame to the plane of the face, $\mathbf{n}$ the unit vector normal to the face, and $\mathbf{u}_{z}$ the unit vector of the $z$ axis of the frame. The line integrals themselves are expressed through a complex set of recurrent relationships that will not be exposed here. The complete set of relationships as well as their derivation can be found in Jamet and Thomas (2004). Similar relationships can be obtained for the normalized spherical harmonic coefficients. The experiments presented in the following make use of the latter formulation.

\section{Assessment}

In order to numerically evaluate the linear algorithm for the computation of polyhedral potential spherical harmonic coefficients, we set up a simple case study consisting of a single tetrahedron of constant density and compare the potential computed from its spherical harmonic expansion to the direct computation with closed analytical formulas (Petrović, 1996). This tetrahedron is the same test body as the one used by Werner (1997).

The geometry of the tetrahedron is shown in figure 1. Each of the four vertices is given by means of its coordinates relative to a local orthogonal reference frame. The origin of the reference frame has been arbitrarily chosen as one of the four vertices. Besides vertex coodinates, the implementation of the linear algorithm requires the knowledge of the vertex topology given as an extra topology matrix with the precondition that the normal vector to each corresponding face will point outside of the tetrahedron. Thus, the two matrices defining respectively the coordinates of the four vertices and their linkage are the following

$$
\mathbf{G}=\left[\begin{array}{rrr}
-2 & -1 & 1 \\
1 & 0 & 1 \\
0 & 1 & 1 \\
0 & 0 & 0
\end{array}\right], \mathbf{H}=\left[\begin{array}{lll}
1 & 2 & 3 \\
1 & 4 & 2 \\
3 & 4 & 1 \\
2 & 4 & 3
\end{array}\right]
$$

Another important parameter in the numerical experiments is the location of the computation point. The expression of the potential in a spherical harmonic expansion implies that the numerical behavior of the series is essentially governed by the powers of the ratio of radii $r_{P} / r_{P}$ where $P$ corresponds to the observation point and $Q$ is a point of the attracting source. It has been theoretically argued that when $r_{P}>r_{Q}$, the series converges for all points located outside a sphere enclosing all masses; for $r_{P}<r_{Q}$, the convergence area is again a sphere, such that the attracting masses are nowhere included or intersected (Tsoulis, 1999). The consequence for the numerical computation of the potential from its spherical harmonic expansion is that a higher degree of expansion using better precision spherical harmonic coefficients is needed for points located close to the attracting source. One should expect conversely that a small 


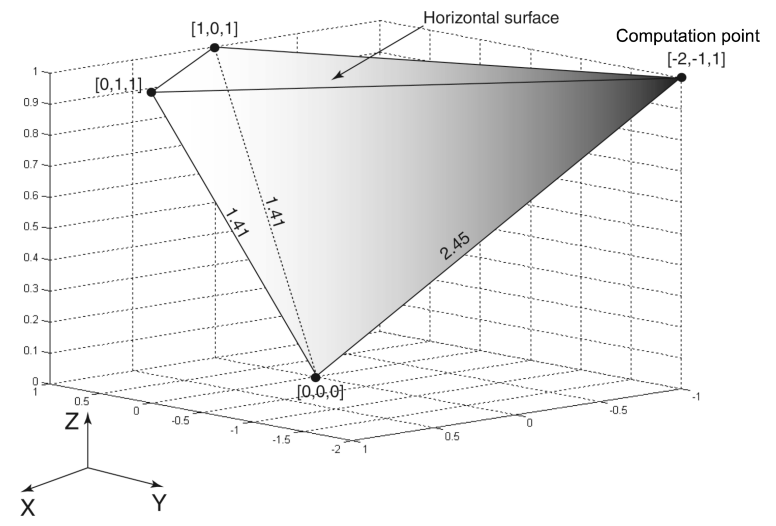

Figure 1: Constant density tetrahedron $\left(5.52 \mathrm{~g} / \mathrm{cm}^{3}\right)$ taken as the attracting source in our numerical experiments. In this case, the upper face of the tetrahedon is horizontal. The farthest point from the origin has been taken as the computation point.

number of spherical harmonic coefficients would be sufficient for reaching the same agreement with the analytical value of the potential when the computation point is located at a large distance from the source. As a result, the best point for performing a numerically significant test of the linear algorithm has to be chosen within the convergence area as close as possible to the source. Thus, the computations in our numerical experiments took place at point $[-2,-1,1]$ of the source tetrahedron where the convergence condition of spherical harmonic series is still satisfied.

Finally, like in Werner (1997), the constant density of the tetrahedral source was chosen equal to $5.52 \mathrm{~g} / \mathrm{cm}^{3}$, which corresponds to an average density value of the Earth's masses.

\subsection{Case of non horizontal faces}

It should be noticed considering the geometry of the tetrahedron used in our experiments that the face formed by vertices numbered 1,2 , and 3 is horizontal, that is parallel to the $x y$ plane of the reference frame. We wanted to investigate in detail whether such a geometry is too much specific and might hide some inherent numerical instabilities when used in the linear algorithm.

The first experiment we carried out for this reason consisted in calculating the potential values provided by the tetrahedra resulting from a rotation around the $x$ axis of the above-mentioned tetrahedron. The potential values were compared to those obtained from a direct computation using closed analytical formulas and the results of the comparison are given in figure 2 . It is clear from this figure that the convergence of the series is ensured when the face of the tetrahedron opposite to the origin is horizontal, which occurs for rotation angles equal respectively to $0^{\circ}$ and $180^{\circ}$. Conversely, for small rotation angles $\left(<35^{\circ}\right.$ or $\left.>135^{\circ}\right)$ the convergence can be only observed at low degrees $(<25)$. For high rotation angles, the divergence immediately occurs except when the tetrahedron face is vertical. In this latter case, specific recursion formulas are used, that do not show the same instabilities. 


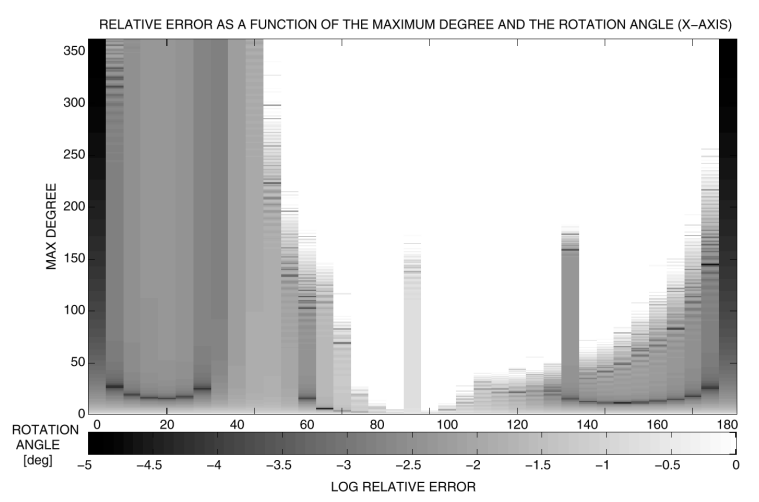

Figure 2: Potential relative error as a function of both the rotation angle and the maximum degree of the spherical harmonic expansion. White indicates when the relative error is greater than $100 \%$.

It is likely that the numerical instabitity observed for a tetrahedron with a tilted face is due to the expression of the recursion fomulas used to calculate the coefficients (eqs. 2 and 3). To put it more precisely, spherical harmonic coefficients of degree $n$ are related to those of degree $n-1$ by the multiplicative factor $\alpha_{n, m}$ in inverse proportion to the cosine of the angle between the outer normal of the tetrahedron face $\mathbf{n}$ and the unit vector $\mathbf{u}_{z}$ of the $z$ axis of the reference frame (eq. 4). This factor increases appreciably when the tilt angle of the tetrahedron face increases, thus causing an exponential growth of the error propagated upon coefficients for increasing degrees. Other possible causes of instabilities that are not discussed here where identified in the recursion process for the computation of the line integrals (unpublished work). They are linked with the coordinates of the leading unit vector of the edges of the tetrahedron and might explain the dissymetry of figure 2 around the rotation angle $90^{\circ}$.

To cope with this instability, an alternative means for computing any tetrahedral source contribution would be to perform the computation in suitable reference frames where the faces involved in the recursion computation remain always horizontal. This method is theoretically justified by the fact that the potential value does not depend on the reference frame used to locate the attracting source and the computation point. From now on, tetrahedral sources with one horizontal face will be only considered.

\subsection{Precision in coefficient computation}

For the purpose of estimating the precision in spherical harmonic coefficient computation by means of the linear algorithm, a computational method to determine the coefficient relative errors was devised and experimented with our case study. Figure 3 shows the relative errors affecting the coefficients, obtained by running the linear algorithm with a random perturbation of the last significant digit of all constant inputs at each step of the recursion and by comparing the results with a computation without perturbation. When using the linear algorithm, the coefficients are calculated vertically from the diagonal by increasing the degree at constant order. The figure indicates that the linear algorithm 


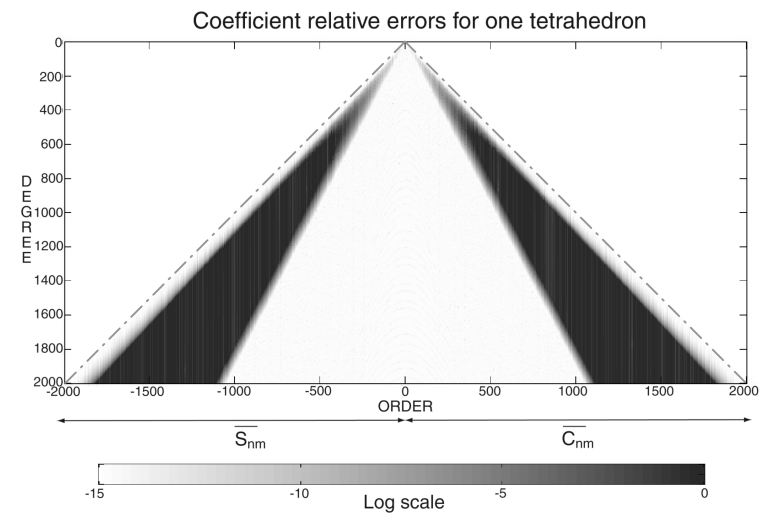

Figure 3: Coefficient relative error as a function of both degree and order. Black indicates when the relative error is greater than $100 \%$.

is stable when the degree increases except near the diagonal where degree and order are similar. The algorithm becomes stable again when the degree $n$ and the order $m$ satisfy $n>1.8 m$.

The maximum degree of the reachable spherical harmonic expansion by means of the linear algorithm can be increased by enlarging the range of the floating-point representation used in the computation. However, once the recursion formulas have become divergent, the coefficient values can no longer be recovered. This is the main limitation inherent to the linear algorithm, which means that some additional procedures should be needed to make the linear algorithm usable in practice at very high degrees.

\subsection{Divergence control}

The last numerical experiment was intented to test the convergence of the linear algorithm for increasing spherical harmonic expansion degrees. Figure 4 shows the relative errors of the cumulated potential as a function of the maximum spherical harmonic degree. These errors have been calculated by comparing the values of the potential provided by the spherical harmonic series deduced from the linear algorithm and those calculated by means of close analytical formulas, thus giving the accuracy of the potential spherical harmonic series for each maximum degree of expansion. The same computation has been carried out with removal of all the coefficients that exhibit a formal relative error greater than $10^{-6}$ as calculated through the method described in subsection 3.2. The potential spherical harmonic series has proven to actually converge up to degree 600. The divergence that occurs afterwards is outstanding, following a quasiexponential growth of the error as the degree increases. The convergence which is obtained, on the other hand, after removing the less precise coefficients is nicely preserved up to degree 1400 where the relative error of the potential determination is somewhat less than $10^{-5}$.

Our findings suggest that the convergence of the potential spherical harmonic expansion resulting from this linear algorithm can be efficiently controlled to ensure the determination of the potential with a reasonable accuracy, which may be sufficient for many applications (terrain effect computation, interpretation of 
Relative error of the cumulated potential at point $[-2,-1,1]$

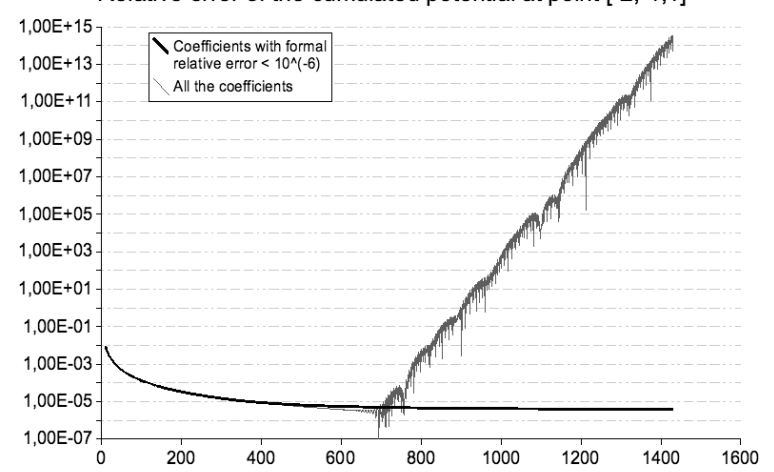

Figure 4: Relative error of the cumulated potential as a function of the maximum degree of the spherical harmonic expansion computed with all the coefficients (gray solid line) and after removing coefficients with a formal error greater than $10^{-6}$ (black thick solid line).

the gravitational signal through comparison of gravity maps, etc.).

\section{Conclusions}

A linear algorithm for computing potential spherical harmonic coefficients of constant density polyhedral sources has been implemented with a simple case study consisting of a single tetrahedron of constant density. The divergence of this algorithm when using a polyhedral source with a non horizontal face opposite to the origin of the frame has been demonstrated conclusively in our first numerical experiment. A change of reference frame which would link each tetrahedron to its horizontal configuration is essential before applying this linear algorithm, which can be readily performed using rotation matrices. The numerical estimation of the coefficient precision has provided the undisputed evidence that the linear algorithm exhibits inherent instabilities affecting the very first terms of the recursion formulas $(n \approx m)$ at high degrees, which would have cast some doubt upon its efficiency. Fortunately, the effect of those numerical instabilities can be completely avoided by removing the coefficients of poor precision without significantly deteriorating the overall potential accuracy. Providing these precautions, the linear algorithm will be empowered to be used for many applications such as, and among others, gravity field and geoid modelling at desired resolution, exact filtering of terrain effects in the computation of residual terrain models or terrain corrections. Moreover, the computation of the potential coefficients generated by real terrestrial bodies will allow us to compare them directly to the coefficients retrieved by gravity exploring satellites such as GOCE. Figure 5 shows an illustration of the ability of the method to map the gravity field at desired resolution and at any location, thus allowing a forward modelling of the gravity sources detectable by GOCE. 


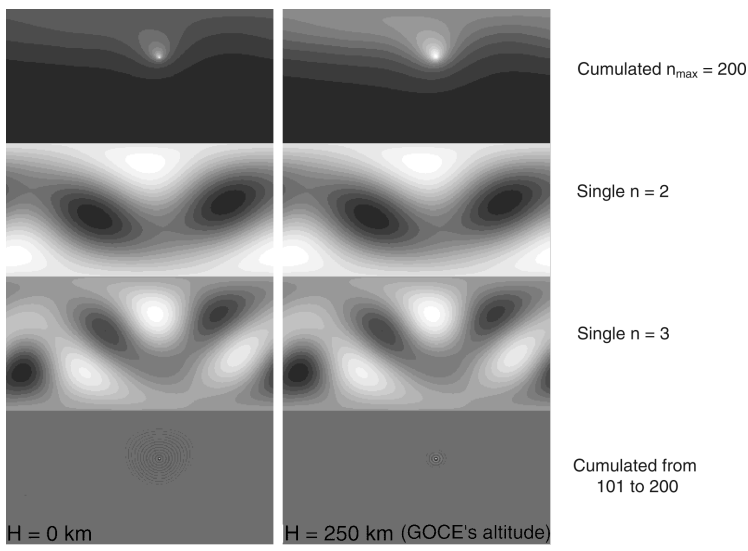

Figure 5: These maps show the global variations of the $T_{z z}$ component of the gravity tensor (latitude and longitude range from respectively $-90^{\circ}$ to $+90^{\circ}$ vertically and $0^{\circ}$ to $360^{\circ}$ horizontally) generated by the tetrahedral source of our case study, and computed on the Earth's surface and at GOCE's altitude $(250 \mathrm{~km})$. The gray scale does not correspond to absolute gravity gradient values, but shows only their relative amplitudes. As expected, the corner which is the closest to the Earth's surface can be revealed by the high degrees of the spherical harmonic expansion (upper and lower maps).

\section{References}

Balmino, G. (1994). Gravitational potential harmonics from the shape of an homogeneous body. Cel Mech Dyn Astr, 60:331-364.

Barnett, C. T. (1976). Theoretical modeling of the magnetic and gravitational fields of an arbitrary shaped three dimensional body. Geophysics, 41:13531364 .

Chao, B. F. and Rubincam, D. P. (1989). The gravitational field of Phobos. Geoph. Res. Let., 16:859-862.

Heiskanen, W. and Moritz, H. (1967). Physical Geodesy. WH Freeman and Company, San Fransisco.

Jamet, O. and Thomas, E. (2004). A linear algorithm for computing the spherical harmonic coefficients of the gravitational potential from a constant density polyhedron. In Proc. Second International GOCE User Workshop, "GOCE, The Geoid and Oceanography", volume ESA SP-569, Frascati, Italy. ESAESRIN.

Martinec, Z., Pěč, K., and Burša, M. (1989). The Phobos gravitational field modeled on the basis of its topography. Earth, Moon and Planets, 45:219 235.

Petrović, S. (1996). Determination of the potential of homogeneous polyhedral bodies using line integrals. J. Geod., 71:44-52. 
Pohánka, V. (1988). Optimum expression for computation of the gravity field of a homogeneous polyhedral body. Geophys. Prosp., 36:733-751.

Simonelli, D., Thomas, P. C., Carcich, B. T., and Vererka, J. (1993). The generation and use of numerical shape models for irregular solar system objects. Icarus, 103:49-61.

Tsoulis, D. (1999). Multipole expressions for the gravitational field of some finite bodies. Bollett. Geod. Sc. A., 58:353-381.

Tsoulis, D. (2001). Terrain correction computations for a densely sampled dtm in the bavarian alps. J. Geod., 75:291-307.

Tsoulis, D. and Petrović, S. (2001). On the singularities of the gravity field of a homogeneous polyhedral body. Geophysics, 66:535-539.

Werner, R. (1997). Spherical harmonic coefficients for the potential of a constant-density polyhedron. Comp. Geosc., 23:1071-1077.

Werner, R. A. and Scheeres, D. J. (1996). Exterior gravitation of a polyhedron derived and compared with harmonic and mascon gravitation representations of asteroid 4769 Castalia. Cel. Mech. Dyn. Astr., 65:313-344. 